\title{
Impulsive Control Approach to Stabilization of Delayed Inertial Neural Networks
}

\author{
R. Krishnasamy \\ \{rksamy123@gmail.com\} \\ Department of Applied Mathematics and Computational Sciences, PSG College of Technology, \\ Coimbatore - 641 004, Tamil Nadu, India.
}

\begin{abstract}
Stabilization of delayed inertial neural networks based on impulsesl is investigated in this paper. Delay-dependent sufficient conditions of stabilization results are obtained as linear matrix inequalities via Lyapunov stability theory which involves the construction of Lyapunov-Krasovskii functional. Information of time-delay is taken into account to obtain these results. Here, time-delay is considered to be time-varying and the activation function is assumed to be sector bounded. Derived conditions can be validated via MATLAB. Finally, an example is provided to support the derived results.
\end{abstract}

Keywords: Inertial neural networks, impulses, Lyapunov-Krasovskii functional, stabilization.

\section{Introduction:}

Past decades witnessed the dynamical analysis of various types of neural networks (NNs) namelyHopfield NNs, bidirectional associative memory (BAM) NNs, cellular NNs, CohenGrossberg NNsand so on. In the year 1981, Babcock and Westervelt published an article on the dynamics of simpleelectronic NNs which initiated the research on inertial NNs (INNs). In their research work, theymentioned that inertial characteristics added to resistance and capacitance couplings can cause spontaneous oscillation, chaotic response and so on. Dynamics of INNs received much attention amongresearchers because of its both physical and biological significance, for details one cansee[1]-[3], [7]-[12] and therein.

Meanwhile, time-delays which may occur during the process of storage of information and transmission in NNscan cause instability, oscillation in the dynamics of NNs. Hence, there is an increasing interest toinvestigate the dynamics of NNs with the inclusion of time-delays, for details see [4]-[8]. Onthe other hand, states of NNs are often condition to abrupt change at certain instants of time dueto the switching phenomenon, frequency change called impulse effects. Impulsive NNs belong to thespecial category which is the combination of continuous and discrete-time systems. Hence, it isnecessary to include the effects of impulses in the dynamics of NNs, see [13]-[15]. Sometimesimpulsive effects can destabilize stable systems which are considered as the destructive one but onthe other hand they can also be used to stabilize the de-stable systems. In this work, impulses areused to stabilize the de-stable systems.

In [13], authors established distributed delay-dependent stability criteria for impulsive INNs inwhich they considered both discrete and distributed time-delays. Robust stability conditions for inertial BAM NNs with time-delays and uncertainties via impulsive effect are considered in [14]. Stabilityconditions of stochastic BAM NNs with Markovian jump,impulse 
control and mixed time-delays is stated in [15]. Hence from the existing literature, it can be seen that stability analysis of INNs withimpulsive effects is taken in to account whereas the problem of stabilization of INNs via impulsivecontrol has not gained attention from the researchers. This motivates us to consider the problem ofimpulsive stabilization of timedelayed INNs.

Inspired by the above observations, stabilization problem of INNs based on impulsive controls isconsidered in this work. Here, the activation function of the neural network is assumed to be sector-bounded and the time-delay is taken to be time-varying. Sufficient delaydependent stabilization results aredeveloped for the considered problem based on the construction ofLyapunov-Krasovskii functional(LKF) involving some quadratic andintegral terms. Schur complement lemma is used in the derivation process to convert some nonlinearmatrix inequalities into linear matrix inequalities (LMIs). Obtained results can be easily solved through MATLAB software.An example is provided to check the validity of the developed theoretical results.

This paper is structured as follows. In second section, the problem under study is formulated and related preliminaries are given. In the third section, sufficient delay-dependent impulsive stabilization conditions pertaining to impulsive control are presented. In the fourth section, a numerical example is given to illustrate the proposed results and the fifth section concludes the paper.

Notations:In the entire paper, $\square^{\mathrm{n}}$ and $\square^{\mathrm{n} \times \mathrm{m}}$ denote, the $\mathrm{n}$-dimensional Euclidean space and the set of all $n \times m$ real matrices respectively. The notation $X \geq Y(X>Y)$ means that $\mathrm{X}-\mathrm{Y}$ is positive-semidefinite (positive-definite), where both $\mathrm{X}$ and $\mathrm{Y}$ represent symmetric matrices; transpose of the matrix $\mathrm{M}$ is denoted by $\mathrm{M}^{\mathrm{T}}$; identity matrix is represented bylwhich is of appropriate dimension and compatible with the corresponding expressions; $\|\cdot\|$ is the Euclidean norm in $\square^{\mathrm{n}} \cdot \operatorname{PC}\left(\left[-\tau_{2}, 0\right], \square^{\mathrm{n}}\right)$ is the piece-wise continuous function;right-hand and left-hand limits of the function $\psi(\mathrm{s})$ are represented by $\psi\left(\mathrm{s}^{+}\right)$and $\psi\left(\mathrm{s}^{-}\right)$respectively; $\lambda_{\max }(\mathrm{A}), \lambda_{\min }(\mathrm{A})$ denote the maximumand minimum eigenvalues of the matrix Arespectively, and for the matrices, if its dimension is not statedexplicitly, aresupposed to have compatible dimensions.

\section{Formulation of the Problem and Preliminaries:}

$$
\ddot{m}_{i}(t)=-a_{i} \dot{m}_{1}(t)-b_{i} m_{i}(t)+\sum_{j=1}^{n} c_{i j} f_{j}\left(m_{j}(t)\right)+\sum_{j=1}^{n} d_{i j} f_{j}\left(m_{j}(t-\tau(t))\right)+I_{i}
$$

for $i=1,2, \cdots, n$, where $\ddot{m}_{i}(t)$ denotes the inertial term of the $i^{\text {th }}$ neuron at time $t, m_{i}(t)$ is the stateof the $\mathrm{i}^{\text {th }}$ neuron at time $t . \mathrm{f}_{\mathrm{j}}(\cdot)$ denotes the neuron activation function of $\mathrm{i}^{\text {th }}$ neuron at time $t$ and $(0)=0, j \in\{1,2, \cdots, n\}, \tau(t)$ is the time-varying delay, $\mathrm{I}_{\mathrm{i}}$ represents the external input on the $i^{\text {th }}$ neuron at time $t . a_{i}$ and $b_{i}$ are positive constants, $c_{i j}$ and $d_{i j}$ are the connection weights related to the neurons without delays and with delays respectively. Initial condition of (1) is given by $\mathrm{m}_{\mathrm{i}}(\mathrm{s})=\varphi_{\mathrm{i}}(\mathrm{s})$ and $\dot{\mathrm{m}}_{\mathrm{i}}(\mathrm{s})=\psi_{\mathrm{i}}(\mathrm{s})$, for $-\tau_{2} \leq \mathrm{s} \leq 0$ where $\varphi_{\mathrm{i}}(\mathrm{s})$ and $\psi_{\mathrm{i}}(\mathrm{s})$ are bounded and continuous.

Now transform the second order differential equation model into a system of first order differentialequations using the transformation $\mathrm{p}_{\mathrm{i}}(\mathrm{t})=\dot{\mathrm{m}}_{1}(\mathrm{t})+\mathrm{m}_{\mathrm{i}}(\mathrm{t}), \mathrm{i}=1,2, \cdots$, nand the resulting system isgiven as

$$
\left\{\begin{array}{c}
\dot{m}_{1}(t)=-m_{i}(t)+p_{i}(t) \\
\dot{p}_{1}(t)=-\left(a_{i}-1\right) p_{i}(t)-\left[b_{i}+\left(1-a_{i}\right)\right] m_{i}(t)+\sum_{j=1}^{n} c_{i j} f_{j}\left(m_{i}(t)\right)+\sum_{j=1}^{n} d_{i j} f_{j}\left(m_{i}(t-\tau(t))\right)+I_{i}
\end{array}\right.
$$


with the initial conditions $\mathrm{m}_{\mathrm{i}}(\mathrm{s})=\varphi_{\mathrm{i}}(\mathrm{s})$ andp $\mathrm{p}_{\mathrm{i}}(\mathrm{s})=\varphi_{\mathrm{i}}(\mathrm{s})+\psi_{\mathrm{i}}(\mathrm{s})$, for $-\tau_{2} \leq \mathrm{s} \leq$ 0. Next, we intend to shift the equillibrium of system (2) to the origin by using the transformation $\mathrm{x}(\mathrm{t})=\mathrm{m}(\mathrm{t})-\mathrm{m}^{*}$ and $\mathrm{y}(\mathrm{t})=\mathrm{p}(\mathrm{t})-\mathrm{m}^{*}$, where $\mathrm{m}(\mathrm{t})=\left[\mathrm{m}_{1}(\mathrm{t}), \mathrm{m}_{2}(\mathrm{t}), \cdots\right.$ , $\left.\mathrm{m}_{\mathrm{n}}(\mathrm{t})\right]^{\mathrm{T}}, \mathrm{p}(\mathrm{t})=\left[\mathrm{p}_{1}(\mathrm{t}), \mathrm{p}_{2}(\mathrm{t}), \cdots, \mathrm{p}_{\mathrm{n}}(\mathrm{t})\right]^{\mathrm{T}}$. Here $\left(\mathrm{m}^{*}, \mathrm{p}^{*}\right)$ is the equillibrium point of (2). Therefore, the transformed system in matrix form canbe written as

$$
\left\{\begin{array}{c}
\dot{x}(\mathrm{t})=-\mathrm{x}(\mathrm{t})+\mathrm{y}(\mathrm{t}) \\
\dot{y}(\mathrm{t})=\mathrm{Ay}(\mathrm{t})-\mathrm{Bx}(\mathrm{t})+\mathrm{Cf}(\mathrm{x}(\mathrm{t}))+\operatorname{Dg}(\mathrm{x}(\mathrm{t}-\tau(\mathrm{t})))
\end{array}\right.
$$

Initial conditions $\mathrm{x}(\mathrm{s})=\phi(\mathrm{s})-\mathrm{m}^{*}$ and $\mathrm{y}(\mathrm{s})=\psi(\mathrm{s})+\phi(\mathrm{s})-\mathrm{p}^{*}$, where $\mathrm{x}(\mathrm{t})=$ $\left[\mathrm{x}_{1}(\mathrm{t}), \mathrm{x}_{2}(\mathrm{t}), \cdots, \mathrm{x}_{\mathrm{n}}(\mathrm{t})\right]^{\mathrm{T}}, \mathrm{y}(\mathrm{t})=\left[\mathrm{y}_{1}(\mathrm{t}), \mathrm{y}_{2}(\mathrm{t}), \cdots, \mathrm{y}_{\mathrm{n}}(\mathrm{t})\right]^{\mathrm{T}}$ are state vectors of $(3), \mathrm{g}(\mathrm{x}(\mathrm{t}))=$ $\left[\mathrm{g}_{1}\left(\mathrm{x}_{1}(\mathrm{t})\right), \mathrm{g}_{2}\left(\mathrm{x}_{2}(\mathrm{t})\right), \cdots, \mathrm{g}_{\mathrm{n}}\left(\mathrm{x}_{\mathrm{n}}(\mathrm{t})\right)\right]^{\mathrm{T}}$ with $\quad \mathrm{g}(\mathrm{x}(\mathrm{t}))=\mathrm{f}\left(\mathrm{x}(\mathrm{t})+\mathrm{m}^{*}\right)-\mathrm{f}\left(\mathrm{m}^{*}\right), \quad \mathrm{A}=$ $\operatorname{diag}\left\{\left(a_{1}-1\right),\left(a_{2}-1\right), \cdots,\left(a_{n}-1\right)\right\}, \quad B=\operatorname{diag}\left\{b_{1}+\left(1-a_{1}\right), b_{2}+\left(1-a_{2}\right), \cdots, b_{n}+\right.$ $\left.\left(1-a_{n}\right)\right\}, C=\left(c_{i j}\right)_{n \times n}, D=\left(d_{i j}\right)_{n \times n}$ and $I=\operatorname{diag}\left\{I_{1}, I_{2}, \cdots, I_{n}\right\}$.

System (3) with continuous and impulsive control becomes

$$
\left\{\begin{array}{c}
\dot{x}(\mathrm{t})=-\mathrm{x}(\mathrm{t})+\mathrm{y}(\mathrm{t})+\mathrm{E}_{1} \mathrm{u}_{1}(\mathrm{t}), \quad \mathrm{t} \neq \mathrm{t}_{\mathrm{k}}, \\
\Delta \mathrm{x}(\mathrm{t})=\left(\mathrm{F}_{1}-\mathrm{I}\right) \mathrm{x}\left(\mathrm{t}^{-}\right)+\mathrm{E}_{2} \mathrm{u}_{2}(\mathrm{t}), \mathrm{t}=\mathrm{t}_{\mathrm{k}}, \\
\dot{y}(\mathrm{t})=\mathrm{Ay}(\mathrm{t})-\mathrm{Bx}(\mathrm{t})+\mathrm{Cf}(\mathrm{x}(\mathrm{t}))+\operatorname{Dg}(\mathrm{x}(\mathrm{t}-\tau(\mathrm{t})))+\mathrm{E}_{3} \mathrm{u}_{3}(\mathrm{t}), \mathrm{t} \neq \mathrm{t}_{\mathrm{k}}, \\
\Delta \mathrm{y}(\mathrm{t})=\left(\mathrm{F}_{2}-\mathrm{I}\right) \mathrm{y}\left(\mathrm{t}^{-}\right)+\mathrm{E}_{4} \mathrm{u}_{4}(\mathrm{t}), \mathrm{t}=\mathrm{t}_{\mathrm{k}},
\end{array}\right.
$$

whereu $u_{1}, \mathrm{u}_{3} \in \mathbb{R}^{\mathrm{n}_{1}}$ are continuous control inputs andu $\mathrm{u}_{2}, \mathrm{u}_{4} \in \mathbb{R}^{\mathrm{n}_{2}}$ are impulse control inputs. $\mathrm{E}_{1}, \mathrm{E}_{2}, \mathrm{E}_{3}, \mathrm{E}_{4}, \mathrm{~F}_{1}$ andF $\mathrm{F}_{2}$ are known constant matrices. $\left.\Delta \mathrm{x}(\mathrm{t})\right|_{\mathrm{t}=\mathrm{t}_{\mathrm{k}}}=\mathrm{x}\left(\mathrm{t}_{\mathrm{k}}^{+}\right)-\mathrm{x}\left(\mathrm{t}_{\mathrm{k}}^{-}\right)$and $\left.\Delta y(t)\right|_{t=t_{k}}=y\left(t_{k}^{+}\right)-y\left(t_{k}^{-}\right) \cdot x\left(t_{k}\right)$ andy $\left(t_{k}\right)$ denote the impulse at the moment $t_{k}$. Here the discrete time sequence $t_{k}$ satisfies $0=t_{0}<t_{1}<t_{2}<\cdots<t_{k}<\cdots$ and $\lim _{k \rightarrow \infty} t_{k}=\infty$. Both $\mathrm{x}\left(\mathrm{t}_{\mathrm{k}}\right)$ and $\mathrm{y}\left(\mathrm{t}_{\mathrm{k}}\right)$ are assumed to be rightcontinuous, i.e., $\mathrm{x}\left(\mathrm{t}_{\mathrm{k}}\right)=\mathrm{x}\left(\mathrm{t}_{\mathrm{k}}^{+}\right)$and $\mathrm{y}\left(\mathrm{t}_{\mathrm{k}}\right)=\mathrm{y}\left(\mathrm{t}_{\mathrm{k}}^{+}\right)$. Initial conditions $\phi(t), \psi(\mathrm{t}) \in \mathrm{PC}\left(\left[-\tau_{2}, 0\right], \mathbb{R}^{\mathrm{n}}\right)$ arepiece-wise continuous functions at finite number of points.

Next design the controllers $\mathrm{u}_{1}, \mathrm{u}_{2}, \mathrm{u}_{3}$ and $\mathrm{u}_{4}$ as follows

$\mathrm{u}_{1}(\mathrm{t})=\mathrm{K}_{1} \mathrm{x}(\mathrm{t}), \mathrm{u}_{2}\left(\mathrm{t}^{-}\right)=\mathrm{K}_{2} \mathrm{x}\left(\mathrm{t}^{-}\right), \mathrm{u}_{3}(\mathrm{t})=\mathrm{K}_{3} \mathrm{y}(\mathrm{t}), \mathrm{u}_{4}(\mathrm{t})=\mathrm{K}_{4} \mathrm{y}\left(\mathrm{t}^{-}\right),(5)$

where $\mathrm{K}_{1}, \mathrm{~K}_{2}, \mathrm{~K}_{3}$ and $\mathrm{K}_{4}$ are control gain matrices to be designed. Also use the fact that $\Delta \mathrm{x}(\mathrm{t})=\mathrm{x}\left(\mathrm{t}_{\mathrm{k}}\right)-\mathrm{x}\left(\mathrm{t}_{\mathrm{k}}^{-}\right)$and $\Delta \mathrm{y}(\mathrm{t})=\mathrm{y}\left(\mathrm{t}_{\mathrm{k}}\right)-\mathrm{y}\left(\mathrm{t}_{\mathrm{k}}^{-}\right)$then system (4) becomes

$$
\left\{\begin{aligned}
\dot{\mathrm{x}}(\mathrm{t}) & =-\mathrm{x}(\mathrm{t})+\mathrm{y}(\mathrm{t})+\mathrm{E}_{1} \mathrm{~K}_{1} \mathrm{x}(\mathrm{t}), \quad \mathrm{t} \neq \mathrm{t}_{\mathrm{k}} \\
\Delta \mathrm{x}(\mathrm{t}) & =\left(\mathrm{F}_{1}-\mathrm{I}\right) \mathrm{x}\left(\mathrm{t}^{-}\right)+\mathrm{E}_{2} \mathrm{~K}_{2} \mathrm{x}\left(\mathrm{t}^{-}\right), \quad \mathrm{t}=\mathrm{t}_{\mathrm{k}} \\
\dot{\mathrm{y}}(\mathrm{t})=\mathrm{Ay}(\mathrm{t})-\mathrm{Bx}(\mathrm{t}) & +\mathrm{Cf}(\mathrm{x}(\mathrm{t}))+\mathrm{Dg}(\mathrm{x}(\mathrm{t}-\tau(\mathrm{t})))+\mathrm{E}_{3} \mathrm{~K}_{3} \mathrm{y}(\mathrm{t}), \quad \mathrm{t} \neq \mathrm{t}_{\mathrm{k}}, \\
\Delta \mathrm{y}(\mathrm{t}) & =\left(\mathrm{F}_{2}-\mathrm{I}\right) \mathrm{y}\left(\mathrm{t}^{-}\right)+\mathrm{E}_{4} \mathrm{~K}_{4} \mathrm{y}\left(\mathrm{t}^{-}\right), \quad \mathrm{t}=\mathrm{t}_{\mathrm{k}} .
\end{aligned}\right.
$$

Next, we give assumptions, definition and lemma which are essential to obtain the results. Assumption 2.1: The neuron activation function $g_{i}(\cdot)$ in (6) is Lipschitz globallyin $x(t), g(0)=0$ and it follows that

$$
\left(g(x(t))-G_{1} x(t)\right)^{T}\left(g(t, x(t))-G_{2} x(t)\right) \leq 0, \quad \forall x(t) \in \mathbb{R}^{n},
$$

where $G_{1}$ and $G_{2}$ are known real constant matrices of appropriate dimensions.

Assumption 2.2: Time-varying delay $\tau(t)$ satisfies

$$
0 \leq \tau(t) \leq \tau_{2}, \quad \dot{\tau}(t) \leq \mu<1,
$$

where $\tau_{2}$ and $\mu$ are constants. 
Definition 2.3: The equilibrium point of INNs with impulsive control and time-delay (6) is said to be exponentially stabilizableunder impulsive control with positive convergence rate $\alpha$ if there exists $\lambda>0$ suchthat

$\|\mathrm{x}(\mathrm{t})\|^{2}+\|\mathrm{y}(\mathrm{t})\|^{2} \leq \lambda \mathrm{e}^{-2 \alpha\left(\mathrm{t}-\mathrm{t}_{0}\right)} \operatorname{Sup}_{-\tau_{2} \leq \mathrm{s} \leq 0}\left[\|\phi(\mathrm{s})\|^{2}+\|\psi(\mathrm{s})\|^{2}\right], \quad \forall \mathrm{t} \geq \mathrm{t}_{0}$.

Definition 2.4: The function $V:[0, \infty) \times \mathbb{R}^{n} \times \mathbb{R}^{n} \rightarrow[0, \infty)$ belong to class $\Omega_{0}$ if

(1) the function $V$ defined above is continuous on all of the sets $\left[t_{k-1}, t_{k}\right) \times \mathbb{R}^{n} \times$ $\mathbb{R}^{\mathrm{n}}, \forall \mathrm{t} \geq 0$ and it vanishes at $\mathrm{V}(\mathrm{t}, 0,0)$,

(2) $V(t, x, y)$ is locally Lipschitzian,

(3) foreveryk $\in \mathbb{N}, \lim _{(t, \bar{x}, \bar{y}) \rightarrow\left(t_{k}^{-}, x, y\right)} V\left(t_{k}^{-}, x, y\right)$ exists and it is finite and further

$\lim _{(t, \bar{x}, \bar{y}) \rightarrow\left(t_{k}^{+}, x, y\right)} V\left(t_{k}^{+}, x, y\right)$ with $V\left(t_{k}, x, y\right)=V\left(t_{k}^{+}, x, y\right)$.

\section{Stabilization results for impulsive INNs:}

In this section, we derive the stabilization conditions for the time-delay INNs based on impulsive control. Here, control gain matrices introduced in (5) is taken as

$\mathrm{K}_{1}=\mathrm{L}_{1} \mathrm{X}^{-1}, \mathrm{~K}_{2}=\mathrm{L}_{2} \mathrm{X}^{-1}, \mathrm{~K}_{3}=\mathrm{L}_{3} \mathrm{Y}^{-1}, \mathrm{~K}_{4}=\mathrm{L}_{4} \mathrm{Y}^{-1}$,

where $L_{1}, L_{2}, L_{3}$ and $L_{4}$ are unknown matrices to be determined.

Theorem: System (6) is exponentially stable under the Assumptions 2.1 and 2.2 if there exist scalars $\alpha, \lambda$, positive-definitesymmetric matrices $\mathrm{P}_{1}, \mathrm{P}_{2}, \widehat{\mathrm{Q}}$ and $\mathrm{R}$, matrices $\mathrm{L}_{1}, \mathrm{~L}_{2}, \mathrm{~L}_{3}$ and $\mathrm{L}_{4}$ suchthat the symmetric LMIs given below hold

$\Omega_{1}<0, \Omega_{2}<0, \Omega_{3}<0$,

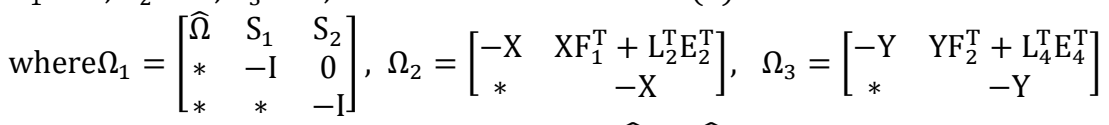

with the stabilizing gains given by (7). Here $\widehat{\Omega}=\left(\widehat{\Omega}_{\mathrm{p}, \mathrm{q}}\right)_{5 \times 5}$ with

$\widehat{\Omega}_{1,1}=-2 \mathrm{X}+\widehat{\mathrm{Q}}+2 \alpha \mathrm{X}+\mathrm{E}_{1} \mathrm{~L}_{1}, \widehat{\Omega}_{1,3}=\mathrm{Y}-\mathrm{XB}^{\mathrm{T}}, \widehat{\Omega}_{1,4}=\mathrm{X}\left(\mathrm{G}_{1}^{\mathrm{T}}+\mathrm{G}_{2}^{\mathrm{T}}\right), \widehat{\Omega}_{\{2,2\}}=$ $-\mathrm{e}^{-2 \alpha \tau_{2}} \widehat{\mathrm{Q}}, \widehat{\Omega}_{3,3}=-2 \mathrm{AY}+2 \alpha \mathrm{Y}+\mathrm{E}_{3} \mathrm{~L}_{3}, \widehat{\Omega}_{3,4}=\mathrm{C}, \widehat{\Omega}_{3,5}=\mathrm{D}, \quad \widehat{\Omega}_{4,4}=\mathrm{R}-2 \mathrm{I}, \widehat{\Omega}_{5,5}=-(1-$

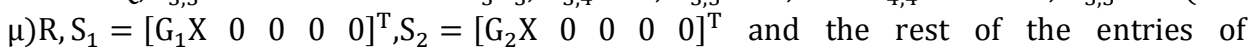
symmetric block are zero.

Proof: Stabilization results for system (6) can be obtained from the construction of LKF as follows

(9)

$$
\begin{gathered}
V(t, x(t), y(t))=x^{T}(t) P_{1} x(t)+y^{T}(t) P_{2} y(t)+\int_{t-\tau_{2}}^{t} e^{2 \alpha(s-t)} x^{T}(s) Q x(s) d s \\
+\int_{t-\tau(t)}^{t} e^{2 \alpha(s-t)} g^{T}(x(s)) \operatorname{Rg}(x(s)) d s
\end{gathered}
$$

where $\mathrm{P}_{1}, \mathrm{P}_{2}, \mathrm{Q}$ and $\mathrm{R}$ are unknown positive-definitesymmetric matrices of appropriate dimensions. From Definition 2.4, one can see that (9) is locally Lipschitz for each $t \in\left[t_{k-1}, t_{k}\right)$ and $V(t, 0,0)=0$. Moreover, all the conditions of Definition 2.4 hold and hence $V(t, x(t), y(t)) \in \Omega_{0}$ for each $t \in\left[t_{k-1}, t_{k}\right)$. Dini's upper right hand derivative of (9) along trajectory of (6) can be determined as follows:

$D^{+} V(t, x(t), y(t)) \leq 2 x^{T}(t) P_{1} \dot{x}(t)+2 y^{T}(t) P_{2} \dot{y}(t)+x^{T}(t) Q x(t)-e^{-2 \alpha \tau_{2}} x^{T}\left(t-\tau_{2}\right) Q x(t$

$$
\left.-\tau_{2}\right)
$$


(10)

$$
\begin{gathered}
+g^{T}(x(t)) \operatorname{Rg}(x(t))-(1-\mu) g^{T} x(t-\tau(t)) \operatorname{Rg}(x(t-\tau(t))) \\
-2 \alpha\left(\int_{t-\tau_{2}}^{t} e^{2 \alpha(s-t)} x^{T}(s) Q x(s) d s+\int_{t-\tau(t)}^{t} e^{2 \alpha(s-t)} g^{T}(x(s)) \operatorname{Rg}(x(s)) d s .\right.
\end{gathered}
$$

According to Assumption 2.1, we have

$$
\left[\begin{array}{c}
\mathrm{x}(\mathrm{t}) \\
\mathrm{g}(\mathrm{x}(\mathrm{t}))
\end{array}\right]^{\mathrm{T}}\left[\begin{array}{cc}
-\left(\mathrm{G}_{1}^{\mathrm{T}} \mathrm{G}_{2}+\mathrm{G}_{2}^{\mathrm{T}} \mathrm{G}_{1}\right) & \mathrm{G}_{1}^{\mathrm{T}}+\mathrm{G}_{2}^{\mathrm{T}} \\
* & -2 \mathrm{I}
\end{array}\right]\left[\begin{array}{c}
\mathrm{x}(\mathrm{t}) \\
\mathrm{g}(\mathrm{x}(\mathrm{t}))
\end{array}\right] \geq 0
$$

Combining inequalities (10) and (11), we get

$\mathrm{D}^{+} \mathrm{V}(\mathrm{t}, \mathrm{x}(\mathrm{t}), \mathrm{y}(\mathrm{t}))+2 \alpha \mathrm{V}(\mathrm{t}, \mathrm{x}(\mathrm{t}), \mathrm{y}(\mathrm{t})) \leq \xi^{\mathrm{T}}(\mathrm{t}) \Omega \xi(\mathrm{t})<0(12)$

which implies that

$\Omega<0$,

$\forall \mathrm{t} \in\left[\mathrm{t}_{\mathrm{k}-1}, \mathrm{t}_{\mathrm{k}}\right)$ where $\xi^{\mathrm{T}}(\mathrm{t})=\left[\begin{array}{lllll}\mathrm{x}^{\mathrm{T}}(\mathrm{t}) & \mathrm{x}^{\mathrm{T}}\left(\mathrm{t}-\tau_{2}\right) & \mathrm{y}^{\mathrm{T}}(\mathrm{t}) & \mathrm{g}^{\mathrm{T}}(\mathrm{x}(\mathrm{t})) & \mathrm{g}^{\mathrm{T}}(\mathrm{x}(\mathrm{t}-\tau(\mathrm{t})))\end{array}\right]$ and $\Omega=\left(\Omega_{1, \mathrm{~m}}\right)_{5 \times 5}$ with

$$
\begin{gathered}
\Omega_{1,1}=-2 \mathrm{P}_{1}+\mathrm{Q}+2 \alpha \mathrm{P}_{1}+\mathrm{P}_{1} \mathrm{E}_{1} \mathrm{~K}_{1}-\left(\mathrm{G}_{1}^{\mathrm{T}} \mathrm{G}_{2}+\mathrm{G}_{2}^{\mathrm{T}} \mathrm{G}_{1}\right), \Omega_{1,3}=\mathrm{P}_{1}-\mathrm{B}^{\mathrm{T}} \mathrm{P}_{2}, \quad \Omega_{1,4}=\mathrm{G}_{1}^{\mathrm{T}}+\mathrm{G}_{2}^{\mathrm{T}}, \\
\Omega_{2,2}=-\mathrm{e}^{-2 \alpha \tau_{2}} \mathrm{Q}, \Omega_{3,3}=-2 \mathrm{P}_{2} \mathrm{~A}+2 \alpha \mathrm{P}_{2}+\mathrm{P}_{2} \mathrm{E}_{3} \mathrm{~K}_{3}, \quad \Omega_{3,4}=\mathrm{P}_{2} \mathrm{C},
\end{gathered}
$$$$
\Omega_{3,5}=\mathrm{P}_{2} \mathrm{D}, \Omega_{4,4}=\mathrm{R}-2 \mathrm{I} \text {, }
$$$$
\Omega_{5,5}=-(1-\mu) \mathrm{R} \text {. }
$$

From (9), at $\mathrm{t}=\mathrm{t}_{\mathrm{k}}$, we have

$$
\begin{aligned}
\mathrm{V}\left(\mathrm{t}_{\mathrm{k}}, \mathrm{x}\left(\mathrm{t}_{\mathrm{k}}\right), \mathrm{y}\left(\mathrm{t}_{\mathrm{k}}\right)\right)- & \mathrm{V}\left(\mathrm{t}_{\mathrm{k}}^{-}, \mathrm{x}\left(\mathrm{t}_{\mathrm{k}}^{-}\right), \mathrm{y}\left(\mathrm{t}_{\mathrm{k}}^{-}\right)\right)=\mathrm{x}^{\mathrm{T}}\left(\mathrm{t}_{\mathrm{k}}\right) \mathrm{P}_{1} \mathrm{x}\left(\mathrm{t}_{\mathrm{k}}\right)+\mathrm{y}^{\mathrm{T}}\left(\mathrm{t}_{\mathrm{k}}\right) \mathrm{P}_{2} \mathrm{y}\left(\mathrm{t}_{\mathrm{k}}\right)-\mathrm{x}^{\mathrm{T}}\left(\mathrm{t}_{\mathrm{k}}^{-}\right) \mathrm{P}_{1} \mathrm{x}\left(\mathrm{t}_{\mathrm{k}}^{-}\right) \\
& \left.=\mathrm{x}^{\mathrm{T}}\left(\mathrm{t}_{\mathrm{k}}^{-}\right) \mathrm{t}_{\mathrm{k}}^{-}\right)\left\{-\mathrm{P}_{1}+\left(\mathrm{K}_{2}^{\mathrm{T}} \mathrm{E}_{2}^{\mathrm{T}}+\mathrm{F}_{1}^{\mathrm{T}}\right) \mathrm{P}_{1}^{-}\left(\mathrm{F}_{1}+\mathrm{E}_{2} \mathrm{~K}_{2}\right)\right\} \mathrm{x}\left(\mathrm{t}_{\mathrm{k}}^{-}\right) \\
& +\mathrm{y}^{\mathrm{T}}\left(\mathrm{t}_{\mathrm{k}}^{-}\right)\left\{-\mathrm{P}_{2}+\left(\mathrm{K}_{4}^{\mathrm{T}} \mathrm{E}_{4}^{\mathrm{T}}+\mathrm{F}_{2}^{\mathrm{T}}\right) \mathrm{P}_{2}\left(\mathrm{~F}_{2}+\mathrm{E}_{4} \mathrm{~K}_{4}\right)\right\} \mathrm{y}\left(\mathrm{t}_{\mathrm{k}}^{-}\right)
\end{aligned}
$$

which implies that

$-\mathrm{P}_{1}+\left(\mathrm{K}_{2}^{\mathrm{T}} \mathrm{E}_{2}^{\mathrm{T}}+\mathrm{F}_{1}^{\mathrm{T}}\right) \mathrm{P}_{1}\left(\mathrm{~F}_{1}+\mathrm{E}_{2} \mathrm{~K}_{2}\right)<0$

$-\mathrm{P}_{2}+\left(\mathrm{K}_{4}^{\mathrm{T}} \mathrm{E}_{4}^{\mathrm{T}}+\mathrm{F}_{2}^{\mathrm{T}}\right) \mathrm{P}_{2}\left(\mathrm{~F}_{2}+\mathrm{E}_{4} \mathrm{~K}_{4}\right)<0$.

One can notice that inequalities (13)-(15) are not LMIs and hence cannot be directly solved by using MATLAB LMI toolbox. So, we make use ofSchur complement lemma to convert the inequalities (14) and (15) as

$$
\left[\begin{array}{cc}
-\mathrm{P}_{1} & \left(\mathrm{~F}_{1}^{\mathrm{T}}+\mathrm{K}_{2}^{\mathrm{T}} \mathrm{E}_{2}^{\mathrm{T}}\right) \mathrm{P}_{1} \\
* & -\mathrm{P}_{1}
\end{array}\right]<0--(\mathrm{a}),\left[\begin{array}{cc}
-\mathrm{P}_{2} & \left(\mathrm{~F}_{2}^{\mathrm{T}}+\mathrm{K}_{4}^{\mathrm{T}} \mathrm{E}_{4}^{\mathrm{T}}\right) \mathrm{P}_{2} \\
* & -\mathrm{P}_{2}
\end{array}\right]<0--(b) .
$$

Now pre- and post-multiply inequalities (16a\&b) respectively by $\operatorname{diag}\{\mathrm{X}, \mathrm{X}\}$ and $\operatorname{diag}\{Y, Y\}$ on both sides and using the relation $X=P_{1}^{-1}$, we get $\Omega_{2}$ and $\Omega_{3}$. Inorder to get $\Omega_{1}$, pre- and post-multiply both sides of (13) by $\operatorname{diag}\{X, I, Y, I, I\}$, where $Y=P_{2}^{-1}$ and also use the relation $-2 \mathrm{X}\left(\mathrm{G}_{1}^{\mathrm{T}} \mathrm{G}_{2}\right) \mathrm{X} \leq \mathrm{XG}_{1}^{\mathrm{T}} \mathrm{G}_{1} \mathrm{X}+\mathrm{XG}_{2}^{\mathrm{T}} \mathrm{G}_{2} \mathrm{X}$. After some algebraic manipulations, one can get $\Omega_{1}$.

From inequality (12), one can get

$V(t, x(t), y(t)) \leq e^{-2 \alpha\left(t-t_{k-1}\right)} V\left(t_{k-1}, x\left(t_{k-1}\right), y\left(t_{k-1}\right)\right)$ for each $t \in\left[t_{k-1}, t_{k}\right) \quad$ and $\mathrm{V}(\mathrm{t}, \mathrm{x}(\mathrm{t}), \mathrm{y}(\mathrm{t})) \leq \mathrm{e}^{-2 \alpha\left(\mathrm{t}-\mathrm{t}_{0}\right)} \mathrm{V}\left(\mathrm{t}_{0}, \mathrm{x}\left(\mathrm{t}_{0}\right), \mathrm{y}\left(\mathrm{t}_{0}\right)\right)$.

From LKF, we get

$\mathrm{V}(\mathrm{t}, \mathrm{x}(\mathrm{t}), \mathrm{y}(\mathrm{t})) \geq \lambda_{1}\left[\|\mathrm{x}(\mathrm{t})\|^{2}+\|\mathrm{y}(\mathrm{t})\|^{2}\right], \quad$ where $\quad \lambda_{1}=\lambda_{\min }\left(\mathrm{P}_{1}\right)+\lambda_{\min }\left(\mathrm{P}_{2}\right)$ and $\mathrm{V}\left(\mathrm{t}_{0}, \mathrm{x}\left(\mathrm{t}_{0}\right), \mathrm{y}\left(\mathrm{t}_{0}\right)\right) \leq \lambda_{2} \operatorname{Sup}_{-\tau_{2} \leq \mathrm{s} \leq 0}\left[\|\phi(\mathrm{s})\|^{2}+\|\psi(\mathrm{s})\|^{2}\right]$, where $\lambda_{2}=\lambda_{\max }\left(\mathrm{P}_{1}\right)+$ $\lambda_{\max }\left(\mathrm{P}_{2}\right)+\tau_{2} \lambda_{\max }(\mathrm{Q})+\tau_{2} \lambda_{\max }(\mathrm{R})$ and hence 
$\|x(t)\|^{2}+\|y(t)\|^{2} \leq \lambda e^{-2 \alpha\left(t-t_{0}\right)} \operatorname{Sup}_{-\tau_{2} \leq s \leq 0}\left[\|\phi(s)\|^{2}+\|\psi(s)\|^{2}\right], \lambda=\frac{\lambda_{2}}{\lambda_{1}}$. Hence by definition of exponential stability, system (6) is stable exponentially with stabilizing gains (7). This completes the proof of the theorem.

Remark: This research work investigates the stabilization analysis of INNs with timedelays under the influence of impulsive controls. Here information of both time-varying delay and its derivative is considered. Even though the stabilization problem of INNs with both discrete and distributed delay under impulsive control is investigated in [13], information on the bound of discrete delay is not considered in [13]. This work is focused to derive stabilization results of INNs which include the data on both time-delay and its derivative.

\section{Numerical Example:}

This section presents a numerical example through which we can see the validity of the derived results.

Example: Consider system (6) with the following parameters

$$
\begin{gathered}
A=\operatorname{diag}\{4,4\}, B=\operatorname{diag}\{12,11\}, C=\left[\begin{array}{cc}
0.3 & 0 \\
-0.6 & -0.4
\end{array}\right], D=\left[\begin{array}{cc}
0.2 & 0.3 \\
0.4 & 0.7
\end{array}\right], \mathrm{E}_{1}=0.3 \mathrm{I}, \mathrm{E}_{2}=0.2 \mathrm{I}, \\
\mathrm{E}_{3}=\mathrm{E}_{4}=0.2 \mathrm{I}, \mathrm{F}_{1}=\mathrm{F}_{2}=0.1 \mathrm{I}, \mathrm{G}_{1}=0.2 \mathrm{I}, \mathrm{G}_{2}=0.1 \mathrm{I}, \tau_{2}=0.6, \mu=0.2, \alpha=0.1,
\end{gathered}
$$

Now, the delay-dependent stabilization conditions obtained in Theorem 3.1 is solved through MATLAB LMI solvers for the above parameters and it can be seen that the considered system (6) is exponentially stabilizable. Feasible matrices that occur in the corresponding conditions of Theorem 3.1 are given below

$$
\begin{aligned}
& \mathrm{X}=\left[\begin{array}{ll}
10.39 & -0.31 \\
-0.31 & 11.26
\end{array}\right], \mathrm{Y}=\left[\begin{array}{cc}
68.92 & -1.68 \\
-1.68 & 68.16
\end{array}\right], \mathrm{Q}=\left[\begin{array}{cc}
37.76 & 0 \\
0 & 37.76
\end{array}\right], \mathrm{R}=\left[\begin{array}{ll}
4.61 & 2.17 \\
2.17 & 7.97
\end{array}\right] . \\
& \text { Stabilizing gains are given by } \\
& \mathrm{K}_{1}=\left[\begin{array}{cc}
-15.32 & -0.38 \\
0.15 & -12.72
\end{array}\right], \mathrm{K}_{2}=\mathrm{K}_{4}=\left[\begin{array}{cc}
-0.5 & 0 \\
0 & -0.5
\end{array}\right], \mathrm{K}_{3}=\left[\begin{array}{cc}
16.65 & 0.02 \\
-0.04 & 16.12
\end{array}\right], \\
& \lambda_{1}=0.10, \lambda_{2}=27.23, \lambda=266.36 \text {. Hence we get } \\
& \quad\|\mathrm{x}(\mathrm{t})\|^{2}+\|\mathrm{y}(\mathrm{t})\|^{2} \leq 266.36 \mathrm{e}^{-0.2 t} \text { Sup }_{-0.6 \leq \mathrm{s} \leq 0}\left[\|\phi(\mathrm{s})\|^{2}+\|\psi(\mathrm{s})\|^{2}\right] \\
& \text { which shows that system (6) is exponentially stabilizable. }
\end{aligned}
$$

\section{Conclusion:}

In this work, stabilizability problem of INNs with time-delay under impulsive control is investigated Both time-varying and constant type of time-delay is taken into account and the corresponding results are presented. LKF involving exponential terms are utilized in the process of obtaining stabilization results. Those conditions are checked using MATLAB through LMI solvers. The problem considered in this research work can be further extended with the incorporation of multiple time-delays in the place of single delay and also with parameter uncertainties.

\section{References:}


[1] J.F. Ashmore, D. Attwell, Models for electrical tuning in hair cells, Proceedings of the Royal Society of London. Series B, Biological Sciences, 226 (1985) 325-344.

[2] K.L. Babcock, R.M. Westervelt, Stability and dynamics of simple electronic neural networks with added inertia, Physica D, 23 (1986) 464-469.

[3] K.L. Babcock, R.M. Westervelt, Dynamics of simple electronic neural networks, Physica D, 28 (1987) 305-316.

[4] P. Balasubramaniam, R. Krishnasamy, Robust Exponential Stabilization Results for Impulsive Neutral Time-Delay Systems with Sector-BoundedNonlinearity, Circuits Systems and Signal Processing, 33 (2014)2741-2759.

[5] K. Gu, L. Kharitonov, J. Chen, Stability of Time Delay Systems, Birkhauser, Boston, 2003.

[6] S. Lakshmanan, C.P. Lim, M. Prakash, S. Nahavandi, P. Balasubramaniam, Neutral-type of delayed inertial neural networks and their stability analysis using the LMI approach, Neurocomputing, 230 (2017) 243-250.

[7] Q. Liu, X. Liao, Y. Liu, S. Zhou, S. Guo, Dynamics of an inertial two-neuron system with time delay, Nonlinear Dynamics, 58 (2009) 573-609.

[8] Y. Liu, W. Liu, L. M.A. Obaid, I.A. Abbas, Exponential stability of Markovian jumping CohenGrossberg neural networks with mixed mode-dependent time-delays, Neurocomputing, 177 (2016) 409-415.

[9] R. Krishnasamy, Raju K. George, Stochastic stability of mode-dependent Markovian jump inertial neural networks, The Journal of Analysis, 27 (2019), 179-196.

[10] R.Krishnasamy, A. Manivannan,Raju K. George, Mean-Square Stochastic Stability of Delayed Hybrid Stochastic InertialNeural Networks, In: Park J. (eds) Recent Advances in Control Problems of Dynamical Systems and Networks. Studies in Systems, Decision and Control, Springer, 301 (2021) 411-433.

[11] M. Prakash, P. Balasubramaniam, S. Lakshmanan, Synchronization of Markovian jumping inertial neural networks and its applications in image encryption, Neural Networks, 83 (2016) 86-93.

[12] R. Rakkiyappan, S. Premalatha, A. Chandrasekar, J. Cao, Stability and synchronization analysis of inertial memristive neural networks with time delays, Cognitive Neurodynamics, 10 (2016) 437451.

[13] T. Yu, H. Wang, M. Su, D. Cao, Distributed-delay-dependent exponential stability of impulsive neural networks with inertial term, Neurocomputing, 313 (2018) 220-228.

[14] W. Zhang, T. Huang, C. Li, J. Yang, Robust stability of inertial BAM neural networks with time delays and uncertainties via impulsive effect, Neural Processing Letters, 48 (2018) 245-256.

[15] Q. Zhu, J. Cao, Stability analysis of Markovian jump stochastic BAM neural networks with impulse control and mixed time delays, IEEE transactions on neural networks and learning systems, 23 (2012) 467-479. 